\title{
Fhit expression in human gastric adenomas and intramucosal carcinomas: correlation with Mlh I expression and gastric phenotype
}

\author{
K Kawaguchi', K Yashima*,1, M Koda', A Tsutsumi', S Kitaoka', H Andachi',2, A Hosoda', Y Kishimoto ${ }^{3}$, \\ G Shiota', $\mathbf{H}$ Ito $^{2}$ and $\mathbf{Y}$ Murawaki'
}

'Division of Medicine and Clinical Science, Faculty of Medicine, Tottori University, Yonago 683-8504, Japan; ${ }^{2}$ Division of Organ Pathology, Faculty of Medicine, Tottori University, Yonago 683-8504, Japan; ${ }^{3}$ Division of Pharmacotherapeutics, Faculty of Medicine, Tottori University, Yonago 683-8504, Japan

\begin{abstract}
The fragile histidine triad (FHIT) gene, encompassing the FRA3B fragile site at chromosome 3p I4.2, is a candidate tumour suppressor gene involved in a variety of tumours, including gastric carcinomas. Recently, it has been reported that the FHIT gene may be a target of damage in some of mismatch-deficient tumours. To clarify further the role of the Fhit protein in gastric carcinogenesis, we investigated whether Fhit expression in early gastric neoplasia is associated with mismatch repair protein expression and cellular phenotype. Fhit, MlhI and phenotypic expression were evaluated immunohistochemically in 87 early gastric neoplasias, comprising 32 adenomas and 55 intramucosal carcinomas, resected by endoscopic mucosal resection therapy. Significant loss or reduction of Fhit expression was noted in four (12.5\%) of the 32 adenomas and 21 (38.2\%) of the 55 intramucosal carcinomas. The rate of abnormal Fhit expression was significantly higher in intramucosal carcinomas than in adenomas $(P=0.02 \mathrm{I})$. Moreover, reduced Fhit expression was found to be significantly associated with loss of Mlh I expression in early gastric neoplasia $(P=0.00 \mathrm{I} I)$. Furthermore, we also detected a significant association between reduced Fhit expression and gastric phenotype $(P=0.00$ I 8$)$. These results suggested that reduced Fhit expression occurs in the early stage of gastric carcinogenesis and could be correlated with a lack of MIh I expression and gastric phenotype.

British Journal of Cancer (2004) 90,672-677. doi: I0.1038/sj.bjc.660 I60 I www.bjcancer.com
\end{abstract}

(c) 2004 Cancer Research UK

Keywords: gastric cancer; Fhit; Mlhl; mucin phenotype; immunohistochemistry; endoscopic mucosal resection

A candidate tumour suppressor gene, fragile histidine triad (FHIT), was identified at chromosome 3p14.2 spanning the FRA3B common fragile site (Ohta et al, 1996). Abnormal transcripts were frequently observed in a variety of human cancers including those of the digestive tract, lung, breast and head and neck (Ohta et al, 1996; Croce et al, 1999). The majority of these abnormalities include aberrant mRNA transcripts, with the absence of one or more exons within the mRNA. Genomic analysis demonstrated frequent allelic loss and homozygous deletions (Ohta et al, 1996; Croce et al, 1999).

In gastric carcinomas, alterations at the FHIT locus or reduced expressions of Fhit have been associated with gastric tumour progression and poor survival of cancer patients (Baffa et al, 1998; Capuzzi et al, 2000). However, conflicting results from reverse transcription (RT) - PCR analysis and genomic analysis have been reported (Ohta et al, 1996; Gemma et al, 1997; Tamura et al, 1997). In general, in tumours (lung and oesophagus) associated with environmental carcinogens, alterations in the FHIT gene occur early in cancer development, but are thought to be a late event in

*Correspondence: K Yashima; E-mail: yashima@grape.med.tottori-u.ac.jp Received 7 July 2003; revised 22 October 2003; accepted 26 November 2003 other cancers and possibly associated with cancer progression to more aggressive neoplasia (Croce et al, 1999; Kitamura et al, 2001). Moreover, little is known about Fhit expression in early gastric neoplasia.

Microsatellite instability (MSI) due to defects in mismatch repair (MMR) genes such as $M L H 1$ and $M S H 2$ are involved in the carcinogenesis and tumour progression of sporadic and inherited human cancers (Eshleman and Makowitz, 1996; Kinzler and Vogelstein, 1996). Microsatellite instability is reportedly present in $15-33 \%$ of solitary gastric cancers, although mutations of the $M L H 1$ or MSH2 genes are rare in sporadic gastric cancers (Chong et al, 1994; Mironov et al, 1994; Strickler et al, 1994; Tamura et al, 1996). Mismatch repair deficiency leads to the accumulation of base-base mismatches and short insertion/deletion mispairs, generated as a consequence of DNA replication errors and homologous recombinations (Eshleman and Makowitz, 1996; Kinzler and Vogelstein, 1996). Most sporadic gastric cancers with MSI have been demonstrated to be caused by somatic hypermethylation of the $M L H 1$ promoter region, resulting in the downregulation of $M L H 1$ gene expression (Fleisher et al, 1999, 2001; Kang et al, 1999; Leung et al, 1999; Suzuki et al, 1999). Recent studies have revealed that immunohistochemistry is a reliable screening technique for identifying MMR-deficient tumours (Thibodeau et al, 1996; Marcus et al, 1999). 
Recently, Fong et al (2000) demonstrated that $N$-nitrosomethylbenzylamine exposure caused a spectrum of visceral and skin tumours similar to Muir-Torre syndrome, caused by deficiency in an MMR gene, in Fhit-deficient mice, and suggested that the FHIT gene may be a target of damage in some of MMR-deficient tumours. Moreover, Mori et al (2001) and Andachi et al (2002) reported that loss of the MMR protein is significantly correlated with the loss of Fhit expression in human colorectal carcinomas. While an association between MSI and FHIT alterations has been reported by Huiping et al (2002), the relationship between Fhit and Mlh1 expression has not been previously studied in gastric carcinomas.

Gastric carcinomas have been divided into two histological types: intestinal and diffuse types, according to Lauren (1965), or differentiated and undifferentiated types, according to Nakamura et al (1968). However, mucin histochemical and immunohistochemical examinations have recently demonstrated that gastric and intestinal phenotypic cell markers are widely expressed in gastric carcinomas, irrespective of their histological type (Egashira et al, 1999; Yoshino et al, 1999; Koseki et al, 2000). Moreover, it has generally been reported that gastric carcinomas with a predominantly gastric phenotype have a pronounced tendency toward invasion, metastasis and poor prognosis compared with gastric carcinomas that have intestinal phenotypic expression (Endo et al, 1999; Koseki et al, 1999; Yoshino et al, 1999; Kabashima et al, 2002; Tajima et al, 2002). Therefore, phenotypic subclassification should be useful in understanding the biologic behaviour of carcinomas and selecting a suitable therapeutic method.

To clarify the role of the FHIT gene in the development of gastric carcinomas, we compared Fhit expression with Mlh1 and phenotypic expression in gastric adenomas and intramucosal carcinomas.

\section{MATERIALS AND METHODS}

\section{Patient samples}

Tumour specimens were obtained from 87 patients (58 male and 29 female subjects), who had undergone endoscopic mucosal resection at Tottori University Hospital between 1994 and 2000. Pathologically, 32 lesions were diagnosed as adenomas and 55 lesions as intramucosal carcinomas (Table 1). Macroscopic and histological evaluations were made according to the classification established by the Japanese Research Society for Gastric Cancer (1993). The macroscopic features were divided into two major types: elevated, and flat or depressed. The depth of invasion and

Table I Clinicopathological features in early gastric neoplasia

\begin{tabular}{|c|c|c|}
\hline & Adenoma $(n=32)$ & $\begin{array}{l}\text { Intramucosal } \\
\text { carcinoma }(n=55)\end{array}$ \\
\hline Gender (M:F) & 18:14 & $40: 15$ \\
\hline Age (mean \pm s.d; years) & $71.3 \pm 6.7$ & $70.0 \pm 8.5$ \\
\hline Histologic type & Mild 2 & Tubi 4I \\
\hline (grade) & Moderate 20 & Tub2 12 \\
\hline & Severe 10 & Pap 2 \\
\hline \multicolumn{3}{|l|}{ Location } \\
\hline Upper & 2 & 4 \\
\hline Middle & 15 & 20 \\
\hline Lower & 15 & 31 \\
\hline \multicolumn{3}{|l|}{ Gross classification } \\
\hline Elevated & 22 & 35 \\
\hline Flat or depressed & 10 & 20 \\
\hline
\end{tabular}

Tubl = well-differentiated tubular adenocarcinoma; Tub2 = moderately differentiated tubular adenocarcinoma; Pap = papillary adenocarcinoma. histological grade were classified according to the predominant features. In this study, adenoma and intramucosal carcinoma correspond to low- or high-grade adenoma/dysplasia and noninvasive carcinoma or intramucosal carcinoma in the Vienna classification system, respectively (Schlemper et al, 2000). Helicobacter pylori infection was evaluated in 20 adenomas and 23 intramucosal carcinomas by histology, rapid urease test, bacterial culture test and serological test, and was defined by a positive result in any of these four tests. All these cases were positive. Pathological diagnoses were verified by two experienced pathologists (HA and HI). All the cases were analysed anonymously, that is, all the specimens were assigned a new number without any personal information. Institutional Review Board approval was obtained.

\section{Immunohistochemical staining}

Paraffin-embedded, $4 \mu \mathrm{m}$-thick sections were immunohistochemically stained with an anti-Fhit rabbit polyclonal antibody (IBL, Gunma, Japan; dilution 1:100), an anti-Mlh1 mouse monoclonal antibody (G168-15, PharMingen, San Diego, CA, USA; dilution $1: 50)$, an anti-human gastric mucin (HGM) mouse monoclonal antibody (45M1, Novocastra Laboratories, Ltd., Newcastle, UK; dilution 1:50), an anti-MUC2 mouse monoclonal antibody (Ccp58, Novocastra, Newcastle, UK; dilution $1: 100$ ) and an anti-CD10 mouse monoclonal antibody (56C6, Novocastra, Newcastle, UK; dilution $1: 50$ ) using the avidin-biotin-peroxidase complex technique.

Immunohistochemical staining was performed as described below. In brief, after being deparaffinised in xylene and rehydrated in ethanol, the sections were immersed in a citrate buffer $(0.01 \mathrm{M}$, $\mathrm{pH}$ 6.0) and heated in a microwave oven for 20-30 min to retrieve antigens, then incubated with the primary antibody overnight at $4{ }^{\circ} \mathrm{C}$. As a negative control, the primary antibody was replaced with normal serum IgG at a similar dilution. The detection reaction followed the Vectastain Elite ABC kit protocol (Vector Laboratories, Burlingame, CA, USA). Diaminobenzidine was used as a chromogen, and methylgreen or haematoxylin as a counterstain. The sections were incubated with biotinylated anti-rabbit or mouse IgG and avidin-biotin-peroxidase and visualised using diaminobenzidine tetrahydrochloride.

Then, paradoxical concanavalin A (ConA, Vector Laboratories, Burlingame, CA, USA) staining was carried out according to the method of Kabashima et al (2002).

The protein expression was evaluated by two independent observers (HA and KY). Immunohistochemical analysis was performed in a blinded manner with respect to the clinical information.

\section{Assessment of Fhit immunostaining}

The Fhit expression was graded for both the extent and intensity of immunopositivity, as described previously (Hao et al, 2000). The extent of positivity was scored as follows: $0,<5 \% ; 1,5-25 \% ; 2$, $25-50 \% ; 3,50-75 \%$ and $4,>75 \%$ of the gastric epithelial cells in the respective lesions. The intensity was scored as follows: 0 , negative; $1+$, weak; $2+$, moderate and $3+$, as strong as normal mucosa. The final score was obtained by multiplying the positivity and intensity scores, producing a range from 0 to 12 . Scores $9-12$ were defined as a preserved or strong staining pattern, scores $5-8$ were defined as an intermediate staining pattern and scores $0-4$ were defined as markedly reduced or lost expression.

\section{Assessment of Mlh1 immunostaining}

Normal tissue adjacent to the tumour was used as an internal positive control. The normal staining pattern for Mlh1 was nuclear. Tumour cells that exhibited an absence of nuclear staining in the 
presence of non-neoplastic cells with nuclear staining were considered to have an abnormal pattern. Cases with definite nuclear staining in more than $30 \%$ of the tumour cells were categorised as positive, cases with definite nuclear staining in less than $30 \%$ of the tumour cells were categorised as negative (Beak et al, 2001).

\section{Assessment of HGM, ConA, MUC2 and CD10 immunostaining and classification of the phenotypes}

Human gastric mucin staining was seen in the cytoplasm of the gastric foveolar epithelium and mucous neck cells, while ConA staining was seen in the cytoplasm of the pyloric glands. MUC2 staining was seen in the cytoplasm around the nuclei of goblet cells. CD10 staining was seen along the brush border of the luminal surface of the epithelium. Although CD10 can also be expressed in the apical portion of the cytoplasm of normal gastric mucosa, only the expression of CD10 on the brush border was studied. The results of staining were categorised into two groups: positive expression and negative expression. Staining of $>10 \%$ of the adenoma and carcinoma cells was classified as positive expression and $<10 \%$ was classified as negative expression. The phenotypes were classified into four categories according to the combination of the expression of CD10, MUC2, ConA and HGM (Yoshino et al, 1999). The intestinal phenotype (I-type) demonstrated positive expression for MUC2 and/or CD10, but negative expression for both HGM and ConA. The gastric and intestinal mixed phenotype (GI-type) demonstrated positive expression for MUC2 and/or CD10, and positive expression for HGM and/or ConA. The gastric phenotype (G-type) demonstrated positive expression for HGM and/or ConA, but negative expression for both MUC2 and CD10. The unclassified phenotype (UC-type) demonstrated negative expression for MUC2, CD10, ConA and HGM.

\section{Statistical analysis}

Statistical analysis was performed by the $\chi^{2}$ test or Fischer's exact test (two-sided). $P<0.05$ was considered significant.

\section{RESULTS}

Fhit expression in the normal epithelium and neoplasia of the stomach

Normal gastric epithelia adjacent to the tumour cells showed moderate to strong cytoplasmic expression of the Fhit protein from the basal portion to the luminal differentiated cells; these findings served as internal positive controls. Smooth muscle cells and inflammatory mononuclear cells were positive at various intensities and degrees. Reduced or absent staining for Fhit was recognised in four (12.5\%) of the 32 adenomas and in $21(38.2 \%)$ of the 55 intramucosal carcinomas (Figure $1 \mathrm{~A}$ and B, Table 2). The incidence of reduced Fhit expression was significantly higher in the intramucosal carcinomas than in the adenomas $(P=0.021)$. However, no significant associations were found among Fhit expression and other clinicopathological parameters (data not shown).

\section{Mlh1 expression in the normal epithelium and neoplasia of the stomach}

The expression of Mlh1 protein was observed exclusively in the nucleus. In normal tissue adjacent to the tumour cells, Mlh1 expression was detected predominantly in the proliferative zone, such as the germinal centres of lymphoid follicles and normal glands. Normal stromal cells such as fibroblasts and endothelial cells also showed nuclear positivity for this protein. Loss of Mlh1 expression was detected in one (3.1\%) of the 32 adenomas and 14
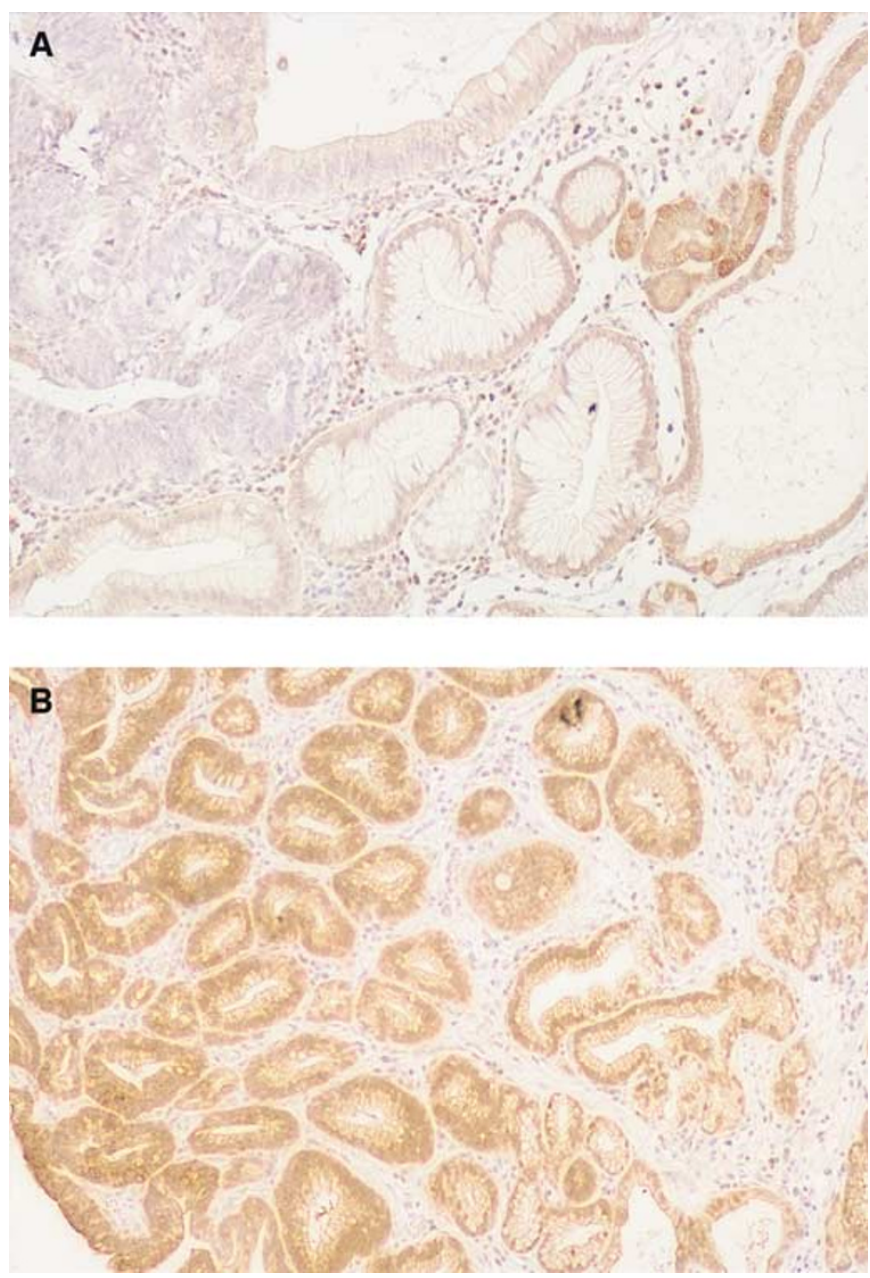

Figure I Fhit immunostaining in human gastric non-neoplastic and neoplastic tissues. (A) Negative immunostaining of an intramucosal carcinoma and positive immunostaining of a non-neoplastic epithelium (B) Positive immunostaining of an adenomatous and non-neoplastic epithelium.

Table 2 Immunohistological findings in early gastric neoplasia

\begin{tabular}{|c|c|c|}
\hline & Adenoma $(n=32)$ & $\begin{array}{l}\text { Intramucosal } \\
\text { carcinoma }(n=55)\end{array}$ \\
\hline \multicolumn{3}{|l|}{ Fhit expression } \\
\hline Preserved or intermediate & 28 & 34 \\
\hline Reduced or absent & 4 & 21 \\
\hline \multicolumn{3}{|l|}{ Mlh1 expression } \\
\hline+ & 31 & 41 \\
\hline - & 1 & 14 \\
\hline \multicolumn{3}{|l|}{ Phenotype } \\
\hline G-type & 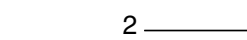 & 16 \\
\hline Gl-type & 10 & 18 \\
\hline I-type & 20 & 19 \\
\hline UC-type & 0 & 2 \\
\hline
\end{tabular}

${ }^{a} P=0.021$, rate of abnormal Fhit expression in adenoma is lower than that in carcinoma. ${ }^{b} P=0.0076$, rate of abnormal Mlh I expression in adenoma is lower than that in carcinoma. ${ }^{c} P=0.013$, rate of G-type in adenoma is lower than that in carcinoma. ${ }^{d} P=0.01 \mathrm{I}$, rate of I-type in adenoma is higher than that in carcinoma.

(25.5\%) of the 55 intramucosal carcinomas (Figure $2 \mathrm{~A}$ and $\mathrm{B}$, Table 2). The incidence of Mlh1 expression loss was significantly higher in the intramucosal carcinomas than in the adenomas 
$(P=0.0076)$. However, there was no significant difference in other clinicopathological parameters (data not shown).

\section{Distribution of the phenotype in early gastric neoplasia}

The distributions of phenotype in the adenomas and intramucosal carcinomas were two $(6.3 \%)$ of 32 and $16(29.1 \%)$ of 55 for the Gtype, $10(31.3 \%)$ of 32 and $18(32.7 \%)$ of 55 for the GI-type, and 20 $(62.5 \%)$ of 32 and $20(36.4 \%)$ of 55 for the I-type, respectively. The frequency of the G-type in carcinomas $(P=0.013)$ and the I-type in adenomas $(P=0.011)$ was significantly higher than in the others (Table 2).

\section{Correlation of Fhit expression with Mlh1 expression in early gastric neoplasia}

Among the early neoplasias with reduced or absent Fhit expression, $10(40.0 \%)$ of 25 showed a loss of nuclear Mlh1 expression, in contrast to five $(8.1 \%)$ of 62 neoplasias with preserved or intermediate Fhit expression $(P=0.0011)$ (Table 3$)$. Regarding intramucosal carcinomas, $42.9 \%$ (nine of 21 ) of the reduced or absent Fhit expression carcinomas were negative for Mlh1 expression, whereas $14.7 \%$ (five of 34 ) of the preserved or intermediate Fhit expression carcinomas were Mlh1 negative
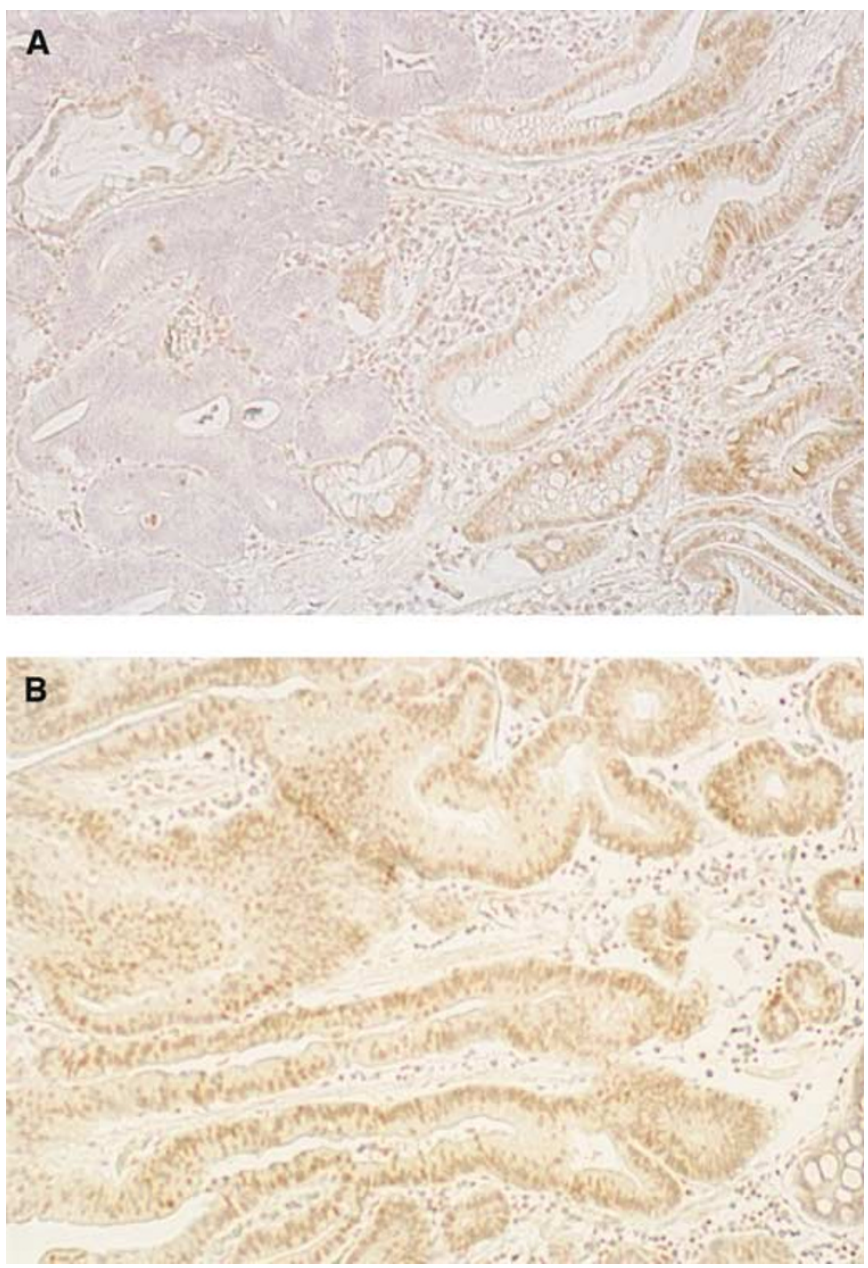

Figure 2 Mlhl immunostaining in human gastric non-neoplastic and neoplastic tissues. (A) Negative immunostaining of an intramucosal carcinoma and positive immunostaining of a non-neoplastic epithelium. (B) Positive immunostaining of an intramucosal carcinoma and nonneoplastic epithelium.
Table 3 Relationship between Fhit and MlhI expression in early gastric neoplasia

\begin{tabular}{lccc} 
& & \multicolumn{2}{c}{ Fhit expression } \\
\cline { 3 - 4 } Mlh lexpression & No. & $\begin{array}{l}\text { Preserved or } \\
\text { intermediate }\end{array}$ & $\begin{array}{l}\text { Reduced or } \\
\text { absent }\end{array}$ \\
\hline+ & 72 & 57 & 15 \\
- & 15 & 5 & 10 \\
\hline
\end{tabular}

$P=0.00 \mathrm{I}$, reduced or absent expression of Fhit is associated with loss of MIh I

Table 4 Relationship between phenotype and Fhit and MIhI expression in early gastric neoplasia

\begin{tabular}{|c|c|c|c|c|c|}
\hline \multirow[b]{2}{*}{ Phenotype } & \multirow[b]{2}{*}{ No. } & \multicolumn{2}{|c|}{ Fhit } & \multicolumn{2}{|c|}{ MIh1 } \\
\hline & & $\begin{array}{l}\text { Preserved or } \\
\text { intermediate }\end{array}$ & $\begin{array}{l}\text { Reduced or } \\
\text { absent }\end{array}$ & + & - \\
\hline G-type & 18 & 7 & $11 \longrightarrow$ & 10 & 8 \\
\hline Gl-type & 28 & 20 & $8 \neg \mathrm{e}$ & 24 & 4 \\
\hline I-type & 39 & 34 & $5 \square$ & 37 & 2 \\
\hline UC-type & 2 & 1 & 1 & 1 & 1 \\
\hline Total & 87 & 62 & 25 & 72 & 15 \\
\hline
\end{tabular}

${ }^{e} P=0.0018$, rate of abnormal Fhit expression in the G-type is higher than that in the others. ${ }^{d} P=0.002$, rate of abnormal Mlh I expression in the G-type is higher than that in the others.

$(P=0.044)$. In the adenomas, the one case with Mlh1-negative expression was one of the four-Fhit-reduced samples.

\section{Relationship between phenotype and expression of Fhit and Mlh1 protein in early gastric neoplasia}

Decreased or nonstaining for Fhit was detected in $11(61.1 \%)$ of 18 G-type, eight $(28.6 \%)$ of 28 GI-type and five (12.8\%) of 39 I-type early gastric neoplasia (Table 4). The incidence of reduced Fhit expression in early gastric neoplasia was significantly more frequent in the G-type compared with the others $(P=0.0018)$. Regarding intramucosal carcinomas, $52.4 \%$ (11 of 21) of the reduced or absent Fhit expression carcinomas were G-type, whereas $14.7 \%$ (five of 34 ) of the preserved or intermediate Fhit expression carcinomas were G-type $(P=0.0073)$. Loss of Mlh1 expression was detected in eight (44.4\%) of $18 \mathrm{G}$ type, four (14.3\%) of 28 GI-type and two (5.1\%) of 39 I-type (Table 4). The incidence of Mlh1 expression loss in early gastric neoplasia was significantly higher in the G-type than in the others $(P=0.0021)$.

\section{DISCUSSION}

Alterations and abnormal transcripts of the FHIT gene have been reported in a number of primary human tumours, including gastric carcinoma (Ohta et al, 1996; Croce et al, 1999). However, some of the data regarding gastric carcinoma are conflicting (Ohta et al, 1996; Gemma et al, 1997; Tamura et al, 1997). It was reported that alterations in the FHIT locus detected by DNA and/or RT PCR analysis closely correlated with a loss of Fhit protein expression in lung and gastric carcinomas (Baffa et al, 1998; Sozzi et al, 1998). These results indicated that FHIT gene alteration could be detected simply by immunohistochemical analysis of tumour specimens. In this study, we observed abnormal Fhit protein expression in intramucosal carcinomas, with $38.2 \%$ of specimens demonstrating a decrease in, or absence of, Fhit protein staining. 
676

This frequency of abnormal Fhit expression was obviously low compared with that observed in advanced gastric carcinomas in previous reports (Baffa et al, 1998; Capuzzi et al, 2000). Therefore, abnormal Fhit expression may be associated with tumour progression in gastric carcinomas. However, we found no correlation between Fhit expression and any of the clincopathological parameters.

Alterations in the FHIT gene and its expression have been reported even in premalignant lesions of the lung, colon and oesophagus (Sozzi et al, 1998; Croce et al, 1999; Kitamura et al, 2001). We found a significantly reduced Fhit expression in $12.5 \%$ of gastric adenomas. However, there was no correlation between Fhit expression and the severity of histopathological changes. Caselli et al (2001) reported that reduced Fhit expression was not detected in precancerous lesions of the stomach. Another recent study on early gastric neoplasia revealed a higher frequency of abnormal Fhit expression comparable to studies on advanced gastric cancer (Skopelitou et al, 2003). These discrepancies may be caused by differences in immunohistochemical techniques, the anti-FHIT antibody used, interpretation of immunoreactivity, sample number, sample type or different distributions of histological grade. Moreover, we found that the rate of reduced Fhit expression was significantly lower in gastric adenomas than that in intramucosal carcinomas. These results suggest that Fhit protein may have a functional role in the early stage of gastric tumorigenesis.

Inactivation of MMR genes has been described as an alternative pathway in cancer development and progression (Eshleman and Makowitz, 1996; Kinzler and Vogelstein, 1996). Microsatellite instability is found in $15-33 \%$ of sporadic gastric cancers, a higher incidence than that seen in other types of sporadic human cancers (Chong et al, 1994; Mironov et al, 1994; Strickler et al, 1994; Tamura et al, 1996). Recent studies have suggested that silencing of the MLH1 gene by promoter hypermethylation is a major causative event in the development of human gastric cancers with MSI (Fleisher et al, 1999, 2001; Kang et al, 1999; Leung et al, 1999; Suzuki et al, 1999). The majority of these tumours also exhibited loss of Mlh1 protein expression. Thus, our finding of loss of Mlh1 expression in 14 $(25.5 \%)$ of the 55 intramucosal carcinomas coincides with published frequencies. However, only one $(3.1 \%)$ of the 32 adenomas showed Mlh1 expression loss. This abnormal Mlh1 protein expression was less frequent than that observed in gastric adenomas reported by other investigators (Beak et al, 2001). This might be partly due to different diagnostic criteria for gastric adenoma, different criteria for the loss of Mlh1 expression, varying degrees of dysplasia and the limited number of tested cases. Moreover, we indicated that the rate of reduced Mlh1 expression in gastric adenomas was significantly lower than that in intramucosal carcinomas, which is consistent with a previous report (Beak et al, 2001).

Recently, it has been reported that following $N$-nitrosomethylbenzylamine exposure, Fhit-deficient mice developed a spectrum of visceral and skin tumours similar to Muir-Torre syndrome, caused by deficiency in an MMR gene (Fong et al, 2000). A large subgroup of Muir-Torre syndrome cases exhibits MSI and germline mutations in the MLH1 or MSH2 gene (Kruse et al, 1998). In addition, it was previously observed that frequent human pancreatic cancers and cell lines with high MSI had homozygous deletions within FHIT (Hilgers and Kern, 1999; Hilgers et al, 2000). Therefore, these reports suggest that the FHIT gene might be a target of damage in some MMR-deficient tumours. Our data show that MMR deficiency based on the status of Mlh1 protein expression is significantly associated with reduced Fhit expression in early gastric neoplasias, supporting this hypothesis. Mori et al (2001) and Andachi et al (2002) reported an association between MMR deficiency and FHIT alterations in colorectal carcinomas. Moreover, as a mechanism for this, they proposed that repetitive elements, such as (CA) $n$ and (A) $n$ repeats, in introns 4 and 5 of the FHIT gene could be a target of damage in MMRdeficient tumours.

It has been reported that there are obvious differences in the biological behaviour of gastric phenotype carcinomas and intestinal phenotype carcinomas (Egashira et al, 1999; Yoshino et al, 1999; Koseki et al, 2000). Generally, gastric phenotype carcinomas are considered to have greater invasiveness and metastatic potential than intestinal phenotype carcinomas (Endo et al, 1999; Koseki et al, 1999; Yoshino et al, 1999; Kabashima et al, 2002; Tajima et al, 2002). In this study, the incidence of reduced or lost Fhit expression in G-type early gastric neoplasias (61.1\%) was significantly higher than that in GI-type $(28.6 \%)$ or I-type $(12.8 \%)$ early gastric neoplasias. Capuzzi et al (2000) reported that the absence of Fhit protein correlated with the progression of gastric carcinomas, higher histological grade and poorer prognosis. Furthermore, MMR-deficient mouse cell lines show increased chromosome fragility (Turner et al, 2002). Therefore, combined analyses of phenotypic expression and Fhit expression by immunohistochemistry could be useful methods for evaluating the malignant potential of gastric neoplasias. In addition, gastric phenotype tumours were associated with a loss of Mlh1 expression. In terms of the relationship between genetic alterations and phenotypes of differentiated-type carcinomas, Endo et al (1999) reported that MSI was detected more frequently in gastric phenotype carcinomas than in intestinal phenotype carcinomas, which is consistent with the present results. Furthermore, we indicated that the incidence of gastric phenotype in gastric adenomas was significantly lower than that in intramucosal carcinomas. This result was also consistent with a previous report (Tsukashita et al, 2001).

In conclusion, we demonstrated reduced Fhit expression in early-stage gastric tumorigenesis, and showed that it was associated with a loss of Mlh1 protein expression and gastric phenotype. Studies that explore the correlation between particular molecular mechanisms and phenotypic expression in neoplasias may offer new insight into gastric carcinogenesis, cancer treatment and feasible chemopreventive pathways.

\section{REFERENCES}

Andachi H, Yashima K, Koda M, Kawaguchi K, Kitamura A, Hosoda A, Kishimoto Y, Shiota G, Ito H, Makino M, Kaibara N, Kawasaki H, Murawaki Y (2002) Reduced Fhit expression is associated with mismatch repair deficiency in human advanced colorectal carcinoma. Br J Cancer 87: $441-445$

Baffa R, Veronese ML, Santoro R, Mandes B, Palazzo JP, Rugge M, Santoro E, Croce CM, Huebner K (1998) Loss of FHIT expression in gastric carcinoma. Cancer Res 58: 4708-4714

Beak MJ, Kang H, Kim SE, Park JH, Lee SJ, Paik YK, Kim H (2001) Expression of hMLH1 is inactivated in the gastric adenomas with enhanced microsatellite instability. Br J Cancer 85: 1147-1152
Capuzzi D, Santoro E, Hauck WW, Kovatich AJ, Rosato FE, Baffa R, Huebner K, McCue PA (2000) Fhit expression in gastric adenocarcinoma. Cancer 88: $24-34$

Caselli M, Marchisio M, Gaudio M, Saragoni L, Lanza G, Alvisi V, Bertagnolo V, Concu M, Capitani S, Caramelli E (2001) Fhit protein expression in human gastric cancer and related precancerous lesions. Oncol Rep 8: $1233-1237$

Chong JM, Fukuyama M, Hayashi Y, Takizawa T, Koike M, Konishi M, Kikuchi-Yanoshita R, Miyaki M (1994) Microsatellite instability in the progression of gastric carcinoma. Cancer Res 54: $4595-4597$ 
Croce CM, Sozzi G, Huebner K (1999) Role of FHIT in human cancer. J Clin Oncol 17: 1618-1624

Egashira Y, Shimoda T, Ikegami M (1999) Mucin histochemical analysis of minute gastric differentiated adenocarcinoma. Pathol Int 49: 55-61

Endo Y, Tamura G, Sakata K, Ohmura K, Watanabe E, Motoyama T (1999) Genetic analysis of differentiated-type adenocarcinomas of the stomach with gastric phenotype and intestinal phenotype (in Japanese with English abstract). Stomach Intestine 34: 539-544

Eshleman JR, Makowitz SD (1996) Mismatch repair defects in human carcinogenesis. Hum Mol Genet 5: 1489-1494

Fleisher AS, Esteller M, Tamura G, Rashid A, Stine OC, Yin J, Zou TT, Abraham JM, Kong D, Nishizka S, James SP, Wilson KT, Herman JG, Meltzer SJ (2001) Hypermethylation of the $h M L H 1$ gene promoter is associated with microsatellite instability in human early gastric neoplasia. Oncogene 20: $329-335$

Fleisher AS, Esteller M, Wang S, Tamura G, Suzuki H, Yin J, Zou TT, Abraham JM, Kong D, Smolinski KN, Shi YQ, Rhyu MG, Powell SM, James SP, Wilson KT, Herman JG, Meltzer SJ (1999) Hypermethylation of the $h M L H 1$ gene promoter in human gastric cancers with microsatellite instability. Cancer Res 59: 1090-1095

Fong LYY, Fidanza V, Zanesi N, Lock LF, Siracusa LD, Mancini R, Siprashvili Z, Ottey M, Martin SE, Druck T, McCue PA, Croce CM, Huebner K (2000) Muir-Torre-like syndrome in Fhit-deficient mice. Pro Natl Acad Sci USA 97: $4742-4747$

Gemma A, Hagiwara K, Ke Y, Burke LM, Khan MA, Nagasima M, Bennett WP, Harris CC (1997) FHIT mutations in human primary gastric cancer. Cancer 57: $1435-1437$

Hao XP, Willis JE, Pretlow TG, Rao JS, MacLnnan GT, Talbot IC, Pretlow TP (2000) Loss of fragile histidine triad expression in colorectal carcinomas and premalignant lesions. Cancer Res 60: 18-21

Hilgers W, Kern SE (1999) Molecular genetic basis of pancreatic adenocarcinoma. Genes Chromosomes Cancer 26: 1-12

Hilgers W, Koerkamp BG, Geradts J, Tang DJ, Yeo CJ, Hruban RH, Kern SE (2000) Genomic FHIT analysis in RER+ and RER- adenocarcinomas of the pancreas. Genes Chromosomes Cancer 27: 239-243

Huiping C, Kristjansdottir S, Bergthorsson JT, Jonasson JG, Magnusson J, Egilsson V, Ingvarsson S (2002) High frequency of LOH, MSI and abnormal expression of FHIT in gastric cancer. Eur J Cancer 38: $728-735$

Japanese Research Society for Gastric Cancer (1993) The General Rules for Gastric Cancer Study, 21st edn Tokyo: Kanehara Publication

Kabashima A, Yao T, Sugimachi K, Tsuneyoshi M (2002) Relationship between biologic behavior and phenotypic expression in intramucosal gastric carcinoma. Hum Pathol 33: 80-86

Kang GH, Shim YH, Ro JY (1999) Correlation of methylation of the $h M L H 1$ promoter with lack of expression of hMLH1 in sporadic gastric carcinomas with replication error. Lab Invest 79: 903-909

Kinzler KW, Vogelstein B (1996) Lessons from hereditary colorectal cancer. Cell 87: $159-170$

Kitamura A, Yashima K, Okamoto E, Andachi H, Hosoda A, Kishimoto Y, Shiota G, Ito H, Kaibara N, Kawasaki H (2001) Reduced Fhit expression occurs in the early stage of esophageal tumorigenesis: no correlation with p53 expression and apoptosis. Oncology 61: 205-211

Koseki K, Takizawa T, Koike M, Funata N, Hishima T, Sakoma T, Moriyama S, Okamoto H, Hayashi S, Iwasaki Y, Arai K. (1999) Subclassification of well differentiated gastric cancer with reference to biological behavior and malignancy, gastric type $v s$ intestinal type, and papillary carcinoma vs tubular carcinoma. Stomach Intestine 34: $507-$ 512

Koseki K, Takizawa T, Koike M, Ito M, Nihei Z, Sugihara K (2000) Distinction of differentiated type early gastric carcinoma with gastric type mucin expression. Cancer 89: 724-732

Kruse R, Rutten A, Lamberti C, Hosseiny-Malayeri HR, Wang Y, Ruelfs C, Jungck M, Mathiak M, Ruzicka T, Hartschuh W, Bisceglia M, Friedl W, Propping P (1998) Muir-Torre phenotype has a frequency of DNA mismatch-repair- gene mutations similar to that in hereditary nonpolyposis colorectal cancer families defined by the Amsterdam criteria. Am J Hum Genet 63: 63-70

Lauren P (1965) The two main histological types of gastric carcinoma: Diffuse and so-called intestinal-type carcinoma. Acta Pathol Microbiol Scand A 64: 31-49

Leung SY, Yuen ST, Chung LP, Chu KM, Chan AS, Ho JC (1999) hMLH1 promoter methylation and lack of hMLH1 expression in sporadic gastric carcinomas with high-frequency microsatellite instability. Cancer Res 59: $159-164$

Marcus VA, Madlensky L, Gryfe R, Kim H, So K, Millar A, Temple LKF, Hsieh E, Hiruki T, Narod S, Bapat BV, Gallinger S, Redston M (1999) Immunohistochemistry for hMLH1 and hMSH2: a practical test for DNA mismatch repair-deficient tumors. Am J Surg Pathol 23: 1248-1255

Mironov NM, Aguelon MA, Potapova GI, Omori Y, Gorbunov OV, Kulimenkov AA, Yamasaki H (1994) Alteration of (CA)n DNA repeats and tumor suppressor genes in human gastric cancer. Cancer Res 54: $41-44$

Mori M, Mimori K, Masuda T, Yoshinaga K, Yamashita K, Matsuyama A, Inoue $\mathrm{H}$ (2001) Absence of Msh2 protein expression is associated with alteration in the FHIT locus and Fhit expression in colorectal carcinoma. Cancer Res 61: 7379-7382

Nakamura K, Sugano H, Takagi K (1968 Carcinoma of the stomach in incipient phase: Its histogenesis and histological appearance. Gann 59: $251-258$

Ohta M, Inoue H, Cotticelli MG, Kastury K, Baffa R, Palazzo J, Siprashvili Z, Mori M, Macue P, Druck T, Croce CM, Huebner K (1996) The FHIT gene, spanning the chromosome 3p14.2 fragile site and renal carcinomaassociated $\mathrm{t}(3 ; 8)$ breakpoint, is abnormal in digestive tract cancers. Cell 84: $587-597$

Schlemper RJ, Riddell RH, Kato Y, Borchard F, Cooper HS, Dawsey SM, Dixon MF, Fenoglio-Preiser CM, Flejou JF, Geboes K, Hattori T, Itabashi M, Iwafuchi M, Iwashita A, Kim YI, Kirchner T, Klimpfinger M, Koike M, Lauwers GY, Lewin KJ, Oberhuber G, Offner F, Price AB, Rubio CA, Shimizu M, Shimoda T, Sipponen P, Solcia E, Stolte M, Watanabe H, Yamabe $H$ (2000) The Vienna classification of gastrointestinal epithelial neoplasia. Gut 47: 251-255

Skopelitou AS, Mitselou A, Katsanos KH, Alexopoulou V, Tsianos EV (2003) Immunohistochemical expression of Fhit protein in Helicobacter pylori related chronic gastritis, gastric precancerous lesions and gastric carcinoma: correlation with conventional clinicopathologic parameters. Eur J Gastroenterol Hepatol 15: 515-523

Sozzi G, Pastorino U, Moiraghi L, Tagliabue E, Pezzella F, Ghirelli C, Tornielli S, Sard L, Huebner K, Pierotti MA, Croce CM, Pilotti S (1998) Loss of Fhit function in lung cancer and preinvasive bronchial lesion. Cancer Res 58: $5032-5037$

Strickler JG, Zheng J, Shu Q, Burgart LJ, Alberts SR, Shibata D (1994) p53 mutations and microsatellite instability in sporadic gastric cancer: when guardians fail. Cancer Res 54: 4750-4755

Suzuki H, Ito F, Toyama M, Kikuchi T, Kakiuchi H, Hinoda Y, Imai K (1999) Distinct methylation pattern and microsatellite instability in sporadic gastric cancer. Int J Cancer 83: 309-313

Tajima Y, Shimoda T, Nakanishi Y, Yokoyama N, Tanaka T, Shimizu K, Saito T, Kawamura M, Kusano M, Kumagai K (2002) Gastric and intestinal phenotypic marker expression in gastric carcinomas and its prognostic significance: immunohistochemical analysis of 136 lesions. Oncology 61: $212-220$

Tamura G, Sakata K, Nishizuka S, Maesawa C, Suzuki Y, Iwaya T, Terashima M, Saito K, Satodate R (1997) Analysis of the fragile histidine triad gene in primary gastric carcinomas and gastric carcinoma cell lines. Genes Chromosomes Cancer 20: $98-102$

Tamura G, Sakata K, Nishizuka S, Maesawa C, Suzuki Y, Terashima M, Eda Y, Satodate R (1996) Allelotype of adenoma and differentiated adenocarcinoma of the stomach. J Pathol 180: 371-377

Thibodeau SN, French AJ, Roche PC, Cunningham JM, Tester DJ, Lindor NM, Moslein G, Baker SM, Liskay RM, Burgart LJ, Honchel R, Halling KC (1996) Altered expression of hMSH2 and hMLH1 in tumors with microsatellite instability and genetic alterations in mismatch repair gene. Cancer Res 56: $4836-4840$

Tsukashita S, Kushima R, Bamba M, Sugihara H, Hattori T (2001) MUC gene expression and histogenesis of adenocarcinoma of the stomach. Int J Cancer 94: $166-170$

Turner BC, Ottey M, Zimonjic DB, Potoczek M, Hauck WW, Pequignot E, Keck-Waggoner CL, Sevignani C, Aldaz CM, McCue PA, Palazzo J, Huebner K, Popescu NC (2002) The Fragile Histidine Triad/Common Chromosome Fragile Site 3B Locus and Repair-deficient Cancers. Cancer Res 62: 4054-4060

Yoshino T, Shimoda T, Saito A, Nakanishi Y, Tajima Y, Shirasu T, Miura S (1999) Macroscopic features of differentiated adenocarcinoma with gastric or intestinal phenotype expression in early gastric cancer (in Japanese with English abstract). Stomach Intestine 34: 513-525 